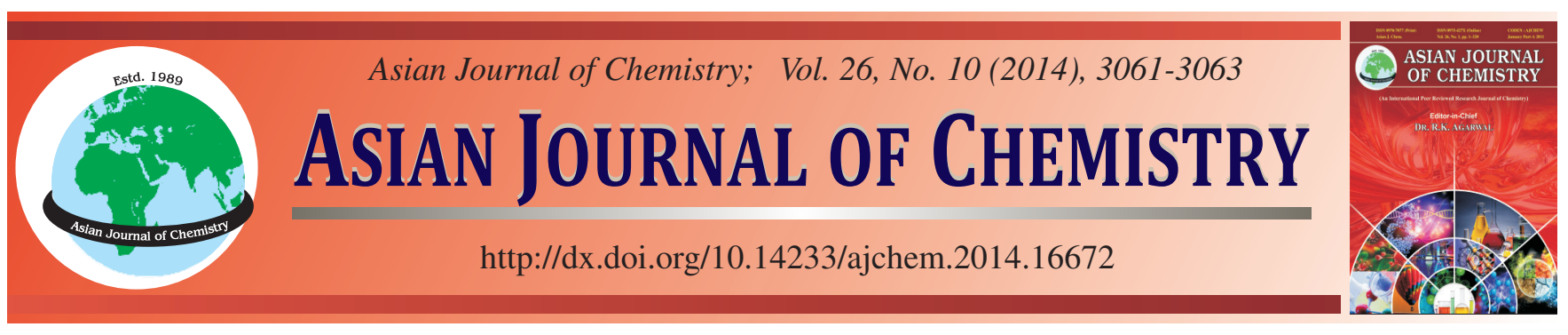

\title{
Anti-Allergic and Antioxidant Effects of Flavonoid Glycosides Isolated from Hop (Humulus lupulus L) Flowers
}

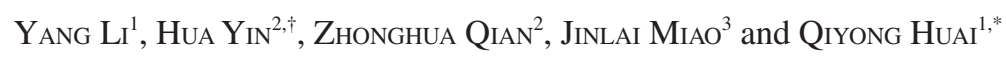

\author{
${ }^{1}$ Marine College, Shandong University, Weihai 264209, P.R. China \\ ${ }^{2}$ State Key Laboratory of Biological Fermentation Engineering of Beer (In Preparation), Qingdao 266061, P.R. China \\ ${ }^{3}$ The First Institute of Oceanography, Qingdao 266061, P.R. China \\ $\dagger$ The author contributed equally to this work
}

*Corresponding author: Tel: +86 13287875796; E-mail: huaiqy01@163.com

Four flavonoid glycosides i.e., isoquercitrin, astragalin, rutin and kaempferol 3-rutinoside, were isolated from the aqueous extract of the hop flowers. In the present study, we carried out the anti-allergic and antioxidant activity of each flavonoid glycosides separately. Isoquercitrin, rutin have the best anti-allergic and antioxidant activity. The structure-activity relationship of flavonoid glycosides for anti-allergic and antioxidant actions were studied. This study suggests that compounds from hop flowers are potential inhibitors against hyaluronidase and antioxidants, indicate a promising strategy for the development of natural anti-allergic, antioxidant medicines and functional foods.

Keywords: Hop flowers, Flavonoid glycosides, Anti-allergic, Antioxidant.

\section{INTRODUCTION}

Hops, a perennial herbaceous vine plant, are widely used for brewing industry, but its medicinal value can not be ignored $^{1}$. Various studies revealed that extracts from hops possess many bioactivities, such as inhibition on bone resorption $^{2}$, anticancer ${ }^{3}$, estrogenic activity ${ }^{4}$ and inhibition on nitric oxide production ${ }^{5}$. Recently, hop water extract, the major fraction of flavonoid glycosides, has been reported to have anti-allergic activity and inhibited histamine release from human basophilic KU812 cells ${ }^{1}$.

Flavonoid glycosides has a wide range of physiological and pharmacological activities, including anti-virus, anti-cancer, anti-inflammatory, anti-allergic and antioxidant effects. Flavonoid glycosides has anti-allergic activity can be used to prevent or alleviate the symptoms such as atopic dermatitis, bronchial asthma, allergic rhinitis, vascular edema, atopic diseases, allergic contact dermatitis, hay fever, urticaria and other allergic diseases. Some studies show that flavonoid glycosides also has antioxidant activity ${ }^{7,8}$, can be used to inhibit the human body oxidation and delay aging.

However, the anti-allergic and antioxidant activity of single flavonoid glycosides from hops haven't been studied before, the relationship between structure and biological activity haven't been mentioned. In this study, we examined the chemical constituents in the flowers of this plant (Tsingdao flower) and isolated four flavonoid glycoside, isoquercitrin (1), astragalin (2), rutin (3), kaempferol 3-rutinoside (4), from the hops water extract (Fig. 1.). Furthermore, we performed a series of experiments to evaluated their hyaluronidase inhibition activity and antioxidant activity in vitro. The relationship between the structure and hyaluronidase inhibition activity, antioxidant activity were investigated.

\section{EXPERIMENTAL}

YMC Gel ODS-A was purchased from Beijing Greenherbs Science and Technology Development Co., Ltd. Amberlite XAD-4, bovine testicular hyaluronidase (EC3.2.1.35, 801 $\mathrm{U} / \mathrm{mg}$ ) and DPPH were purchased from Sigma-Aldrich Chemical Co. (St. Louis, MO, USA). Hyaluronic acid sodium salt was the product of Solarbio Ltd. (Beijing, China) and other reagents were reagent grade available and purchased from J\&K Chemical Ltd. (Beijing, China). The absorbance were measured on a UV-754 spectrophotometer (Shanghai Analytical Instrument Factory). The $1 \mathrm{H}$ spectra were recorded on a Bruker AMX400 NMR spectrometer.

Hops flowers were provided by Tsingtao Brewery Group (Qingdao, China).

Extracion and isolation: Air-dried, powdered flowers $(1 \mathrm{~kg})$ of Hops were extracted with cold water $\left(10 \mathrm{~L}, 5^{\circ} \mathrm{C}\right.$, $24 \mathrm{~h}$ ). The cold water extract was transferred to a separatory funnel, successively extraction by petroleum ether (one time, 


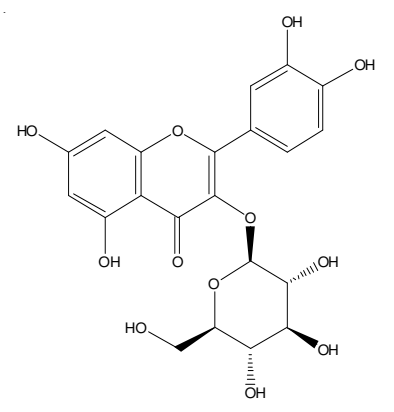

1

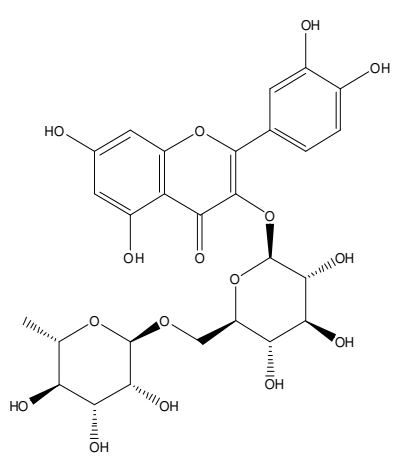

3

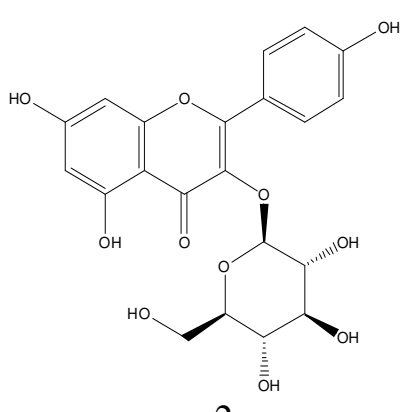

2

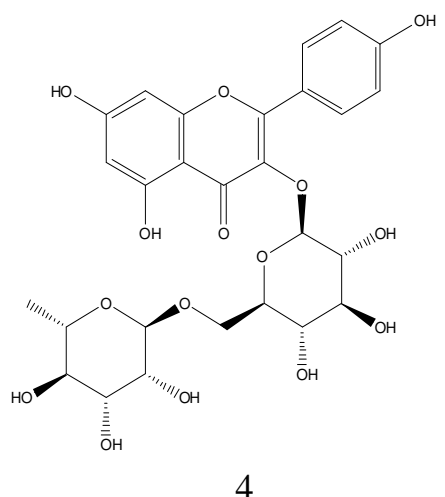

4
Fig. 1. Chemical structures of compounds 1-4

petroleum ether-migrating components were discarded), ethyl acetate (three times, ethyl acetate-migrating components were discarded) and 1-butanol (one time, 1-butanol-migrating components were retain). The butanol layers were concentrated under reduced pressure to obtain extract, dissolved the extract by water and concentrated it. Then apply it to Amberlite XAD-4, the column was eluted with distilled water, 30, 50, 70 and $95 \%$ ethanol stepwise. $50 \%$ ethanol fraction was chromatographed over a $\mathrm{C}-18$ reversed-phase column to obtain compounds 1 (3 mg), 2 (3.2 mg), 3 (3.8 mg) and 4 (3.6 mg).

Assessment inhibition against hyaluronidase activity: Effect on hyaluronidase enzyme activity was measured by the modified Morgan-Elson method with slight modifications ${ }^{9}$. Briefly, hyaluronidase enzyme $(1250 \mathrm{U} / \mathrm{mL})$ and calcium chloride $(2.5 \mathrm{mmol} / \mathrm{L})$ were incubated for $20 \mathrm{~min}$ at $37{ }^{\circ} \mathrm{C}$. Then, the isolated compounds was added to the mixture and again incubated for $40 \mathrm{~min}$ at $37{ }^{\circ} \mathrm{C}$. The hyaluronic acid sodium solution $(0.5 \mathrm{mg} / \mathrm{mL})$ was added and incubation at 37 ${ }^{\circ} \mathrm{C}$ for $40 \mathrm{~min}$. After the addition of sodium hydroxide and acetylacetone, the reaction mixture was heated in a boiling water bath for $10 \mathrm{~min}$ to stop the enzyme reaction. After cooling to room temperature, Ehrlich's reagent was added and incubated at $37^{\circ} \mathrm{C}$ for $20 \mathrm{~min}$ when color developed. The absorbance at $530 \mathrm{~nm}$ of the clear supernatant was measured. Hyaluroni- dase inhibition activity was expressed by decreased percentage as compared with control.

To evaluate the anti-allergic activity of the compounds extracted from hop flowers, we investigated their inhibitory effect on hyaluronidase and found that these compounds showed different levels of inhibition against hyaluronidase. As shown in Table-1, over a range of 50-200 $\mu \mathrm{M}$, isoquercitrin, astragalin, rutin, kaempferol 3-rutinoside showed obvious inhibition against hyaluronidase, with the inhibition rate of $59.23 \pm 4.39,53.85 \pm 6.08,65.38 \pm 3.85$ and $58.46 \pm 4.21 \%$ at $200 \mu \mathrm{M}$, respectively. It has been reported that, in KU812 cell, isoquercitrin, astragalin, rutin, kaempferol 3-rutinoside could inhibit the release of histamine, which is a key chemical mediator in the allergic disease ${ }^{6}$.

Assessment radical-scavenging activity by DPPH method: Radical-scavenging activity of several flavonoid glycosides was analysed by the DPPH method ${ }^{10}$. The samples were prepared by dissolving each compound in DMSO. These samples were assayed at different concentrations with suitable dilutions, making them up to $1 \mathrm{~mL}$, followed by addition of $1 \mathrm{~mL}$ of 2,2-diphenyl-1-picrylhydrazyl (400 $\mu \mathrm{M}$ solution in ethanol) and mixing of the contents by vigorous shaking. The tubes were incubated in the dark at room temperature for 20 min. The absorbance was then recorded at $517 \mathrm{~nm}$, using DMSO as a blank.

Radical-scavenging activity $(\%)=[($ Control OD-sample OD)/control OD] $\times 100 \%$. These experiments were carried out in quintuplicate.

\section{RESULTS AND DISCUSSION}

These flavonoid glycosides showed different levels of inhibition against hyaluronidase. As shown in Table-1, over a range of 50-200 $\mu \mathrm{M}$, compounds 1-4 showed obvious inhibition against hyaluronidase, with the inhibition rate of 59.23 $\pm 4.39,53.85 \pm 6.08,65.38 \pm 3.85$ and $58.46 \pm 4.21 \%$ at 200 $\mu \mathrm{M}$, respectively. The anti-allergic activity were decreased in the order of $\mathbf{3}>\mathbf{1}>\mathbf{4}>\mathbf{2}$. The quercetin type of flavonol glycosides was found to have higher inhibitory activity than kaempferol glycoside. 3'-OH and glycoside can enhance the anti-allergic activity.

But in the antioxidant activity were decreased in the order of $\mathbf{3}>\mathbf{1}>\mathbf{2}>\mathbf{4}$. Again, amongst the quercetin glycosides showed stronger activity than the kaempferol derivatives, indicating the importance of a catechol grouping in the B-ring in enhancing the antioxidant activity.

Flavonoid glycosides may be useful as a functional food component or an alternative medicine for the relief of allergy symptoms and retardation of senescence and this report will help to estimate antiallergic and antioxidant effects based on

TABLE-1

INHIBITORY EFFECT OF COMPOUNDS ISOLATED FROM HOP FLOWERS ON HYALURONIDASE AND DPPH

\begin{tabular}{|c|c|c|c|c|c|c|}
\hline \multicolumn{7}{|c|}{ Inhibition $(\% \text { control })^{\mathrm{a}}$} \\
\hline \multirow{2}{*}{ Compound } & \multicolumn{3}{|c|}{ Hyaluronidase } & \multicolumn{3}{|c|}{ DPPH } \\
\hline & $50 \mu \mathrm{M}$ & 100 & $200 \mu \mathrm{M}$ & $50 \mu \mathrm{M}$ & $100 \mu \mathrm{M}$ & $200 \mu \mathrm{M}$ \\
\hline Isoquercitrin & $32.31 \pm 3.44$ & $45.38 \pm 5.01$ & $59.23 \pm 4.39$ & $29.31 \pm 1.21$ & $52.10 \pm 0.99$ & $54.15 \pm 1.22$ \\
\hline Astragalin & $23.85 \pm 3.22$ & $38.46 \pm 4.71$ & $53.85 \pm 6.08$ & $2.47 \pm 0.91$ & $5.33 \pm 0.89$ & $5.55 \pm 0.84$ \\
\hline Rutin & $43.08 \pm 5.70$ & $56.15 \pm 5.83$ & $65.38 \pm 3.85$ & $32.86 \pm 0.83$ & $54.30 \pm 0.67$ & $57.18 \pm 0.38$ \\
\hline Kaempferol 3-rutinoside & $26.92 \pm 2.72$ & $42.31 \pm 6.08$ & $58.46 \pm 4.21$ & $0.97 \pm 1.18$ & $2.15 \pm 1.24$ & $3.32 \pm 1.48$ \\
\hline
\end{tabular}


the composition of flavonoid glycosides in food materials. Those results would be utilized as valuable guide for the development of new anti-allergic and antioxidant drugs.

\section{ACKNOWLEDGEMENTS}

This study was supported by the Open Research Fund of State Key Laboratory of Biological Fermentation Engineering of Beer (in preparation) under grant No. K2012007 and Natural Science Foundation of Shandong Province (No. ZR2010BM021).

\section{REFERENCES}

1. J.F. Stevens and J.E. Page, Phytochemistry, 65, 1317 (2004).

2. H. Tobe, Y. Muraki, K. Kitamura, O. Komiyama, Y. Sato, T. Sugioka, H.B. Maruyama, E. Matsuda and M. Nagai, Biosci. Biotechnol. Biochem., 61, 158 (1997).
3. P.J. Magalhaes, D.O. Carvalho, J.M. Cruz, L.F. Guido and A.A. Barros, Nat. Prod. Commun., 4, 591 (2009).

4. S. Milligan, J. Kalita, V. Pocock, A. Heyerick, L. De Cooman, H. Rong and D. De Keukeleire, Reproduction, 123, 235 (2002).

5. F. Zhao, H. Nozawa, A. Daikonnya, K. Kondo and S. Kitanaka, Biol. Pharm. Bull., 26, 61 (2003).

6. S. Segawa, K. Yasui, Y. Takata, T. Kurihara, H. Kaneda and J. Watari, Biosci. Biotechnol. Biochem., 70, 2990 (2006).

7. C.N.A. Leong, M. Tako, I. Hanashiro and H. Tamaki, Food Chem., 109, 415 (2008).

8. Z. Zhao, J. Jin, J. Ruan, C. Zhu, C. Lin, W. Fang and Y. Cai, J. Nat. Prod., 70, 1683 (2007).

9. K. Ippoushi, Y. Yamaguchi, H. Itou, K. Azuma and H. Higashio, Food Sci. Technol. Res., 6, 74 (2000).

10. O.P. Sharma and T.K. Bhat, Food Chem., 113, 1202 (2009). 